\title{
Clinical Translation of Ex Vivo Sentinel Lymph Node Mapping for Colorectal Cancer Using Invisible Near-Infrared Fluorescence Light
}

\author{
Merlijn Hutteman, MSc ${ }^{1,2}$, Hak Soo Choi, PhD $^{2}$, J. Sven D. Mieog, MD ${ }^{1}$, Joost R. van der Vorst, MD ${ }^{1}$, \\ Yoshitomo Ashitate, $\mathbf{M D}^{2}$, Peter J. K. Kuppen, $\mathbf{P h D}^{1}$, Marian C. van Groningen, MD, $\mathbf{P h D}^{3}$, \\ Clemens W. G. M. Löwik, PhD ${ }^{4}$, Vincent T. H. B. M. Smit, MD, PhD ${ }^{3}$, Cornelis J. H. van de Velde, MD, PhD ${ }^{1}$, \\ John V. Frangioni, MD, $\mathbf{P h D}^{2,5}$, and Alexander L. Vahrmeijer, MD, PhD $^{1}$ \\ ${ }^{1}$ Department of Surgery, Leiden University Medical Center, Leiden, The Netherlands; ${ }^{2}$ Division of Hematology/Oncology, \\ Department of Medicine, Beth Israel Deaconess Medical Center, Boston, MA; ${ }^{3}$ Department of Pathology, Leiden \\ University Medical Center, Leiden, The Netherlands; ${ }^{4}$ Department of Endocrinology, Leiden University Medical Center, \\ Leiden, The Netherlands; ${ }^{5}$ Department of Radiology, Beth Israel Deaconess Medical Center, Boston, MA
}

\begin{abstract}
Background. Sentinel lymph node (SLN) mapping in colorectal cancer may have prognostic and therapeutic significance; however, currently available techniques are not optimal. We hypothesized that the combination of invisible near-infrared (NIR) fluorescent light and ex vivo injection could solve remaining problems of SLN mapping in colorectal cancer.

Methods. The FLARE imaging system was used for realtime identification of SLNs after injection of the NIR lymphatic tracer HSA800 in the colon and rectum of $(n=4)$ pigs. A total of 32 SLN mappings were performed in vivo and ex vivo after oncologic resection using an identical injection technique. Guided by these results, SLN mappings were performed in ex vivo tissue specimens of 24 consecutive colorectal cancer patients undergoing resection.

Results. Lymph flow could be followed in real-time from the injection site to the SLN using NIR fluorescence. In pigs, the SLN was identified in 32 of 32 (100\%) of SLN mappings under both in vivo and ex vivo conditions. Clinically, SLNs were identified in all patients $(n=24)$ using the ex vivo strategy within $5 \mathrm{~min}$ after injection of fluorescent tracer. Also, 9 patients showed lymph node
\end{abstract}

(C) The Author(s) 2010. This article is published with open access at Springerlink.com

First Received: 16 April 2010; Published Online: 16 November 2010

A. L. Vahrmeijer, MD, PhD

e-mail: a.l.vahrmeijer@lumc.nl involvement ( $\mathrm{N} 1$ disease). In 1 patient, a 3-mm mesenteric metastasis was found adjacent to a tumor-negative SLN. Conclusions. The current pilot study shows proof of principle that ex vivo NIR fluorescence-guided SLN mapping can provide high-sensitivity, rapid, and accurate identification of SLNs in colon and rectum. This creates an experimental platform to test optimized, non-FDA-approved NIR fluorescent lymphatic tracers in a clinical setting.

The prognosis and quality of life for patients suffering from colorectal cancer (CRC) depends on the extent and quality of surgical treatment. Apart from tumor characteristics (i.e., stage, differentiation grade), complete surgical removal with en bloc regional lymphadenectomy is pivotal for patient prognosis. Metastatic spread to regional lymph nodes is one of the most important prognostic factors and determines the need for adjuvant chemotherapy. The sentinel lymph node (SLN) is the first lymph node that receives lymphatic drainage from a tumor, and identification of the SLN and analysis for tumor involvement should predict the status of the remaining lymph nodes. The SLN procedure is regarded standard of care in the treatment of breast cancer and melanoma. ${ }^{1-3}$ However, its added value in colorectal cancer has not yet been established.

Retrospective studies on micrometastatic disease in CRC and the risk of recurrence are conflicting. ${ }^{4-6}$ Our group has previously shown that ultrastaging of lymph nodes with RT-PCR identifies a subgroup of $\mathrm{pN} 0$ patients with an inferior prognosis. ${ }^{7,8}$ However, this methodology is labor intensive and not used routinely. Identifying the SLN 
in the surgical specimen provides the pathologist with an opportunity to optimally stage the lymphatic basin beyond standard H\&E analysis of the regional nodes. ${ }^{9}$ Published results on identifying SLN in CRC show variable success rates ranging from $58 \%$ to $100 \% .{ }^{10-20}$ Consequently, there is ample room for improvement of mapping methodology.

In the majority of published studies of CRC patients, subserosal, circumferential, and peritumoral injection of the visible dye isosulfan blue has been used, both in vivo and ex vivo. ${ }^{12,13,15,19,21}$ Isosulfan blue is distributed rapidly through the afferent lymphatic channels; however, because of their small size, the dye particles can readily diffuse through the true SLN to 2nd- and 3rd-tier nodes (a total of up to 21 "sentinel" nodes are reported). ${ }^{16}$ Isosulfan blue also stains tissue an unnatural color and provides poor overall contrast.

Dyes that emit fluorescence in the invisible, near-infrared (NIR) portion of the electromagnetic spectrum $(700-900 \mathrm{~nm})$ have several advantages for SLN mapping. NIR light penetrates relatively deep into living tissue, and tissue itself has low autofluorescence in the NIR, resulting in a high signal-tobackground ratio. ${ }^{22}$ Since NIR light is not visible to the human eye, there is no change to the look of the surgical field. Several clinical studies have been published on SLN mapping in breast cancer, gastric cancer, and colon cancer, using the clinically available NIR probe indocyanine green. ${ }^{23-27}$ However, these studies were performed using imaging systems that display the NIR signal without the visible image as a surgical anatomical reference. Our group has developed a system (FLARE) that is capable of displaying NIR signal and visible image (captured by a color video camera) simultaneously and can superimpose the NIR signal over the color image. ${ }^{28,29}$

Previously, our group reported the use of NIR light for real-time intraoperative SLN mapping of the gastrointestinal tract. ${ }^{30,31}$ These and earlier studies show clear advantages of NIR imaging over the conventional blue dyes. ${ }^{32}$ Of published organic molecules, the NIR fluorophore HSA800 emerged as the molecule with the best overall performance with respect to entry to the lymphatics, flow to the SLN, retention in the SLN, fluorescence yield, and reproducibility. Since HSA800 cannot be translated to the clinic without a long and costly regulatory process, we hypothesized that ex vivo SLN mapping in colon and rectal cancer could provide all of the benefits of NIR fluorescence imaging over visible blue dyes, without the need for regulatory approval, and without risk to the patient.

\section{MATERIALS AND METHODS}

\section{Lymphatic Tracers}

The preparation of HSA800 (IRDye 800CW conjugated to human serum albumin) has been described in detail previously. ${ }^{30}$ The ratio of fluorophore (IRDye $800 \mathrm{CW}$; Li-Cor, Lincoln, NE) to albumin (HSA) was 3:1, estimated using the extinction coefficients of HSA $\left(\varepsilon_{280 \mathrm{~nm}}=32,900\right.$ $\left.\mathrm{M}^{-1} \mathrm{~cm}^{-1}\right)$ and CW800 $\left(\varepsilon_{778 \mathrm{~nm}}=240,000 \mathrm{M}^{-1} \mathrm{~cm}^{-1}\right)$ in phosphate-buffered saline (PBS). Peak absorbance and emission of HSA800 were 778 and $795 \mathrm{~nm}$, respectively. A stock solution of $10 \mu \mathrm{M}$ (in pig) or $50 \mu \mathrm{M}$ (in human) HSA800 in PBS, pH 7.4 was used for all injections.

\section{Real-Time NIR Fluorescence Imaging}

Preclinical SLN mapping was performed using the FLARE image-guided surgery system as described in detail previously. ${ }^{29}$ The system is shown schematically in Fig. 1. It consists of 2 wavelength isolated light sources: a "white" light source, generating 40,000 lx of 400-650 nm light and a "near-infrared" light source, generating 12.5$\mathrm{mW} / \mathrm{cm}^{2} 760-\mathrm{nm}$ light over a $15-\mathrm{cm}$ field of view. Color video and NIR fluorescence images are simultaneously acquired and displayed in real time using custom optics and software that separate the color video and NIR fluorescence images. ${ }^{29}$ A pseudocolored (lime green) merged image of the color video and NIR fluorescence images is also displayed. Clinical SLN mapping was performed using a miniaturized version of FLARE (Mini-FLARE), which is described in detail elsewhere (Troyan et al., manuscript in preparation), with settings of 20,000 lx of $400-650 \mathrm{~nm}$ "white" light and $7 \mathrm{~mW} / \mathrm{cm}^{2}$ of $760-\mathrm{nm}$ NIR light.

\section{Animal Model Systems}

Animals were housed in an AAALAC-certified facility staffed by full-time veterinarians and were studied under the supervision of an approved institutional protocol. This protocol adhered to the "Guide for the Care and Use of Laboratory Animals," NRC 1996. Four female Yorkshire pigs were purchased at $35 \mathrm{~kg}$ from E.M. Parsons and Sons (Hadley, MA). All animals acclimated to the animal facility for at least $48 \mathrm{~h}$ prior to experimentation. Anesthesia was induced with $4.4-\mathrm{mg} / \mathrm{kg}$ intramuscular Telazol (Fort Dodge Labs, Fort Dodge, IA). The animal was then intubated, and anesthesia was maintained with $2 \%$ isoflurane (Baxter Healthcare Corporation, Deerfield, IL). Electrocardiogram (ECG), heart rate, oxygen saturation, and body temperature were monitored throughout the experiment. At the end of each experiment, euthanasia was induced by rapid intravenous injection of $10 \mathrm{~mL}$ of Fatal-Plus (Vortech Pharmaceuticals, Dearborn, MI), a method consistent with the recommendation of the Panel on Euthanasia of the American Veterinary Medical Association. 
FIG. 1 FLARE image-guided surgery system. The dichroic mirror directs light below and above $650 \mathrm{~nm}$ to a color video camera and NIR fluorescence camera, respectively, permitting simultaneous acquisition of color video and NIR fluorescence images. The surgeon's monitor includes a pseudocolored (lime green) merge of the 2 images

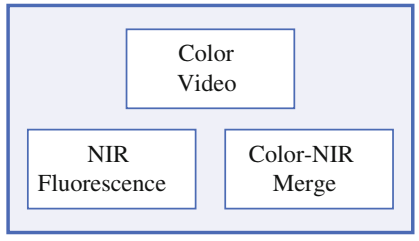

Surgeon's Monitor

White Light (400-650 nm)

NIR Fluorescence Excitation (745-779 nm)

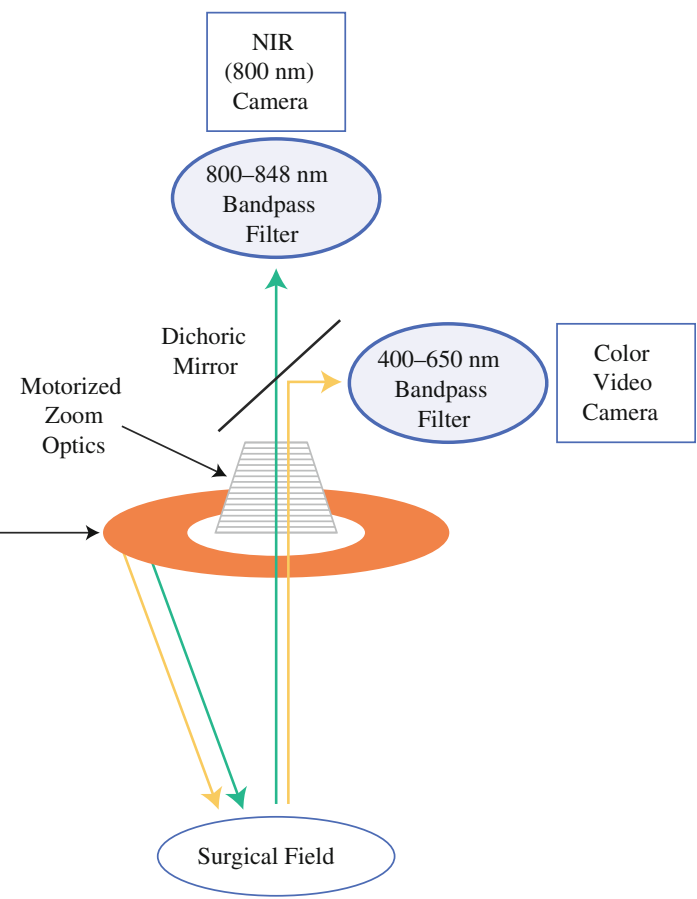

\section{Preclinical Sentinel Lymph Node Mapping In Vivo Versus Ex Vivo}

SLN mapping was performed using a 1-cc syringe equipped with a 27-gauge, 0.5-in. needle. Each injection consisted of $0.1 \mathrm{cc}$ of HSA800 solution injected subserosally, with the needle bevel facing upward. The injection site was then gently massaged to stimulate lymphatic flow through increased hydrostatic pressure.

All animals received multiple injections at separate locations in the colon and rectum. For each condition (in vivo and ex vivo), 8 injections were performed in colon and 8 injections were performed in the rectum, for a total of 32 injections. The NIR fluorescence signal was monitored using the FLARE system, and the SLN identified by watching uninterrupted lymphatic flow reach the first lymph node in real-time. After successful identification, the suspected lymph node was resected under fluorescence guidance, fixed in $2 \%$ paraformaldehyde for $12 \mathrm{~h}$, frozen in $\mathrm{LN}_{2}$ using Tissue-Tek OCT, cryosectioned at $10 \mu \mathrm{m}$, and analyzed by hematoxylin and eosin (H\&E) staining.

After the in vivo injections, a subtotal colectomy and a total mesorectal excision (TME) were performed. The resected bowel specimens were placed on a table and at locations that were not injected in vivo, $0.1 \mathrm{cc}$ of HSA800 was injected subserosally, identical to the technique used for in vivo injections. Tissue samples were also collected and processed for histology as described previously.

Time needed to identify the SLN was compared between in vivo and ex vivo conditions using an unpaired $t$ test. Assumption of equal variances was confirmed by Levene's test. Statistical analyses, performed using SPSS software version 17.0 (SPSS Inc., Chicago, IL), were 2-tailed, and $P<.05$ was considered significant.

\section{Translation to the Clinic}

A total of 24 consecutive patients undergoing curativeintended surgery for colorectal cancer were included. This study was approved by the Leiden University Medical Center Medical Ethics Committee and performed in accordance with the ethical standards of the Helsinki Declaration of 1975. All patients underwent a standard oncological resection including lymphadenectomy. In cases of colon cancer, a segmental colonic resection was performed; in cases of rectal cancer, a resection following the principles of the total mesorectal excision (TME) was performed. Directly following resection, bowel segments were delivered fresh to the department of pathology, where the specimen was opened anti-mesenterically by the pathologist. In rectal cancer, surgical quality audit protocol prohibits altering the perirectal fat in any way. Consequently, in rectal cancer, the specimen was opened no further than the rectosigmoid junction. Then, $1 \mathrm{cc}$ of $50-\mu \mathrm{M}$ HSA800 was injected submucosally circumferentially with a 5-mm margin around the tumor, and the injection site was massaged for $5 \mathrm{~min}$. After $5 \mathrm{~min}$, the specimen was inspected using Mini-FLARE. Any fluorescent hotspots were either marked with a suture or directly resected for separate fixation in $2 \%$ buffered formalin. The specimen was fixed using $2 \%$ buffered formalin for $24 \mathrm{~h}$ (colon) or $48 \mathrm{~h}$ (rectum). After fixation, fluorescent nodes 
were identified under direct NIR fluorescence guidance with the Mini-FLARE, and the specimen was processed following the standard clinical protocol. For rectal cancer, the specimen was processed using the slicing technique as described by Quirke et al. ${ }^{33}$ All lymph nodes (sentinel and nonsentinel) were paraffin embedded and $4-\mu \mathrm{m}$ sections were $\mathrm{H} \& \mathrm{E}$ stained and subsequently analyzed by microscope for the presence of tumor cells.

\section{RESULTS}

\section{Preclinical NIR Fluorescence-Guided SLN Identification and Resection}

In both in vivo and ex vivo conditions, all injections led to a successful identification of a SLN (Table 1). Shortly after massaging the injection site, lymphatic flow was visualized on the NIR fluorescence image (Fig. 2). In all cases, lymphatic flow terminated, without interruption, in 1 or more lymph nodes.

In the colon, we found no difference (Table 1) in the time needed to identify the SLN between in vivo (56.4 seconds on average) and ex vivo (58.8 seconds on average) conditions $(t=-0.111, P=.913)$. In the rectum, however, ex vivo injection led to a significantly faster identification time for the SLN (26.8 seconds on average) compared with in vivo (54.1 seconds; $t=2.942$, $P=.011)$. Mean time between resection of the bowel and ex vivo injection was $25 \mathrm{~min}$ (range 10-57 min). All resected specimens $(n=32)$ were analyzed by $\mathrm{H} \& \mathrm{E}$ histology and confirmed to be lymph node tissue (Fig. 2c).

\section{Clinical Ex Vivo NIR Fluorescence-Guided SLN Mapping in Colorectal Cancer Patients}

Patient and tumor characteristics are listed in Table 2.

In a preliminary set of experiments on $n=6$ clinical specimens, it was found that the subserosal injection technique that worked so well with normal pig specimens did not provide reliable results with human tumor specimens (data not shown). Injecting subserosally and circumferentially around human tumors resulted in a larger injection site, which outshined lymph nodes close to the tumor. To overcome this problem, a submucosal injection technique was used. ${ }^{16,34}$ It was also found that because of the thickness of human specimens versus normal pig specimens, a high concentration $(50 \mu \mathrm{M})$ of HSA800 was optimal.

Using this optimized strategy, in all 24 consecutive patients, the procedure led to a successful detection of SLNs. An example of the NIR fluorescence images of the procedure in colon cancer is shown in Fig. 3a. In rectal cancer, quality control dictates that the specimen is not altered prior to inking, fixation, and slicing. As shown in Fig. 3b, in rectal cancer, nodes were harvested successfully after mesorectal slicing. Based on prefixation and postfixation images, the formalin fixation process did not appear to alter NIR fluorescence of HSA800. On average, per specimen, 3.0 SLNs (range 1-5 SLNs) were identified by NIR fluorescence (Table 2). On average, a total of 15.9 lymph nodes (range 8-31 lymph nodes) were harvested per specimen. Histological analysis showed that 9 of 24 patients had lymph node metastases. In all but 1 of those cases, at least 1 of the SLNs contained tumor cells. In 1 patient, however, a small tumor deposit $(3 \mathrm{~mm})$ was found in the mesentery, whereas the SLN was tumor negative. Although no clear lymphatic tissue could be identified (Fig. 4), pathological guidelines (WHO Classification, 6th edition) require that this lesion be classified as a lymph node metastasis.

\section{DISCUSSION}

The presence of lymph node metastases in CRC patients is the most important prognostic factor and an indication for adjuvant chemotherapy. The presence of nodal involvement decreases 5-year survival rates by $25-35 \%$. Long-term follow-up of adjuvant chemotherapy for patients with stage III colon cancer showed a survival benefit with a $22-33 \%$ relative reduction in mortality. ${ }^{35,36}$ Therefore, adequate staging in order to select patients who may benefit from adjuvant chemotherapy treatment is a necessity. Moreover, patients without nodal disease (stage I/II) still develop recurrent tumors in up to $25 \%$ of the cases
TABLE 1 Preclinical in vivo and ex vivo identification rates for SLN mapping of pig colon and rectum with HSA800

\begin{tabular}{cllll}
\hline Organ & $\begin{array}{l}\text { Number of } \\
\text { injections }(n)\end{array}$ & $\begin{array}{l}\text { Successful } \\
\text { identifications, } n(\%)\end{array}$ & $\begin{array}{l}\text { Number of SLNs } \\
\text { identified, } n\end{array}$ & $\begin{array}{l}\text { Average identification } \\
\text { time, sec }\end{array}$ \\
\hline $\begin{array}{c}\text { Colon } \\
\text { In vivo }\end{array}$ & 8 & & & \\
Ex vivo & 8 & $8(100 \%)$ & 9 & $56.4 \pm 48.3$ \\
Rectum & $8(100 \%)$ & 8 & $58.8 \pm 36.2$ \\
In vivo & 8 & $8(100 \%)$ & & $54.1 \pm 16.3$ \\
Ex vivo & 8 & $8(100 \%)$ & 10 & $26.8 \pm 21.3$ \\
\hline
\end{tabular}


FIG. 2 In vivo and ex vivo SLN mapping in colon and rectum. a Identification of the SLN (arrows) in pig colon after in vivo (top row) or ex vivo (bottom row) injection (arrowheads) of $0.1 \mathrm{cc}$ of $10 \mu \mathrm{M}$ HSA800. Shown are the fluorescence (middle), and pseudocolored (lime green) merge of the 2 (right). b Ex vivo identification of the SLN (arrow) after injection (arrowhead) of $0.1 \mathrm{cc}$ of $10 \mu \mathrm{M}$ HSA800 (top row). Resected negative $(-)$ and positive $(+)$ nodes in rectum (bottom row). Shown are the color video (left), NIR fluorescence (middle), and pseudocolored (lime green) merge of the 2 (right). Note accumulation of the dye at the sinus entry site. c H\&E

histological staining of the $(+)$ tissue section $(100 \times$

magnification) from $\mathbf{b}$ color video (left), NIR
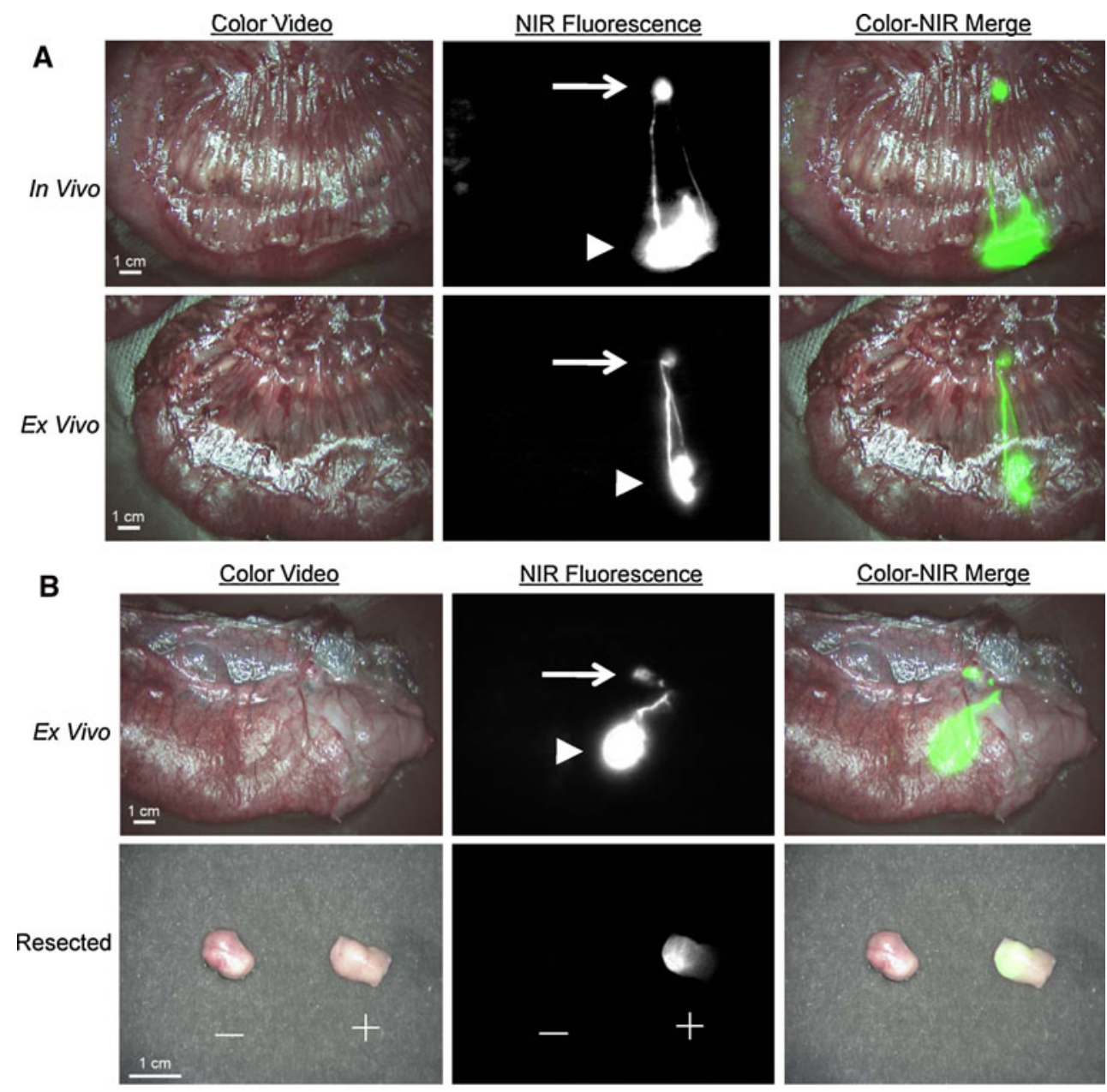

C

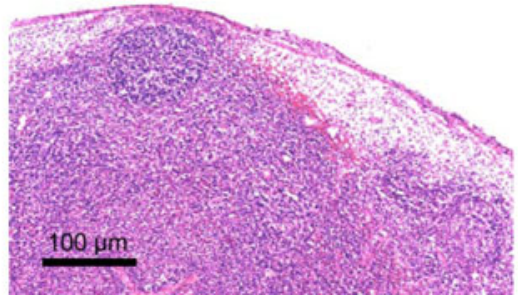

within 5 years after surgical resection of the primary tumor. This could be due to pathological understaging of the tissue specimen at the time of primary treatment. SLN mapping would be a useful method to circumvent this problem. $^{12}$

Contradicting studies have also been published, showing a high false negative rate of SLN mapping in colon cancer. $^{37,38}$ These so-called skip metastases, a tumor-negative SLN combined with a positive non-SLN, are an important limiting factor to the validity of the SLN procedure. In colorectal cancer, skip metastases rates of $3-60 \%$ are reported. ${ }^{12,19}$ It has been suggested that these variable rates are caused by the applied SLN identification technique, which could be overcome by a more reliable method of identifying the SLN.
The current study shows proof of principle that by using invisible NIR fluorescent light and an optimal contrast agent, identification of the lymphatic channels and the SLN is rapid and accurate. In the presented preclinical experiments in pigs, both ex vivo NIR fluorescence imaging of the SLN as well as in vivo imaging showed similar results. These preclinical results were successfully translated into a pilot clinical study in which the SLN was identified ex vivo in all patients. Whereas the NIR fluorescent signal of subserosal injections outshined lymph nodes located close to the tumor, submucosal injections showed superior results in patient specimens.

In 1 patient with three tumor-negative SLNs, a small tumor metastasis was found in the mesenteric fat, without any remnants of pre-existent lymphatic tissue (Fig. 4). 
TABLE 2 Patient characteristics and results of sentinel lymph node mapping

\begin{tabular}{|c|c|c|c|c|c|c|c|c|c|c|c|}
\hline Patient & $\begin{array}{l}\text { Age } \\
\text { (year) }\end{array}$ & $\begin{array}{l}\text { Body } \\
\text { mass } \\
\text { index }\end{array}$ & $\begin{array}{l}\text { Tumor } \\
\text { location }\end{array}$ & $\begin{array}{l}\text { Tumor } \\
\text { differentiation }\end{array}$ & $\begin{array}{l}\text { Tumor } \\
\text { size } \\
(\mathrm{cm})\end{array}$ & $\begin{array}{l}\text { Tumor } \\
\text { stage }\end{array}$ & $\begin{array}{l}\text { NIR- } \\
\text { identified } \\
\text { SLNs }\end{array}$ & $\begin{array}{l}\text { Tumor } \\
\text { positive } \\
\text { SLNs }\end{array}$ & $\begin{array}{l}\text { Total } \\
\text { LN }\end{array}$ & $\begin{array}{l}\text { Total } \\
\mathrm{LN}+\end{array}$ & $\begin{array}{l}\text { False } \\
\text { negative }\end{array}$ \\
\hline 1 & 80 & 24 & Cecum & Moderately & 6.0 & $\mathrm{~T} 3$ & 2 & 0 & 16 & 0 & No \\
\hline 2 & 69 & 29 & Cecum & Moderately & 5.5 & $\mathrm{~T} 3$ & 3 & 0 & 20 & 0 & No \\
\hline 3 & 66 & 21 & Cecum & Moderately & 6.7 & $\mathrm{~T} 3$ & 3 & 0 & 18 & 0 & No \\
\hline 4 & 38 & 39 & Cecum & Moderately & 3.5 & $\mathrm{~T} 4$ & 2 & 0 & 20 & 0 & No \\
\hline 5 & 92 & 27 & Cecum & Moderately & 4 & $\mathrm{~T} 3$ & 4 & 2 & 12 & 3 & No \\
\hline 6 & 65 & 16 & Cecum & Moderately & 1.3 & $\mathrm{~T} 2$ & 5 & 0 & 12 & 0 & No \\
\hline 7 & 68 & 21 & Ascending colon & Moderately & 5 & $\mathrm{~T} 4$ & 5 & 1 & 24 & 5 & No \\
\hline 8 & 67 & 35 & Ascending colon & Moderately & 4 & $\mathrm{~T} 3$ & 3 & 0 & 31 & 0 & No \\
\hline 9 & 75 & 26 & Ascending colon & Moderately & 4 & $\mathrm{~T} 3$ & 3 & 0 & 11 & 0 & No \\
\hline 10 & 67 & 37 & Ascending colon & Moderately & 2.3 & $\mathrm{~T} 2$ & 2 & 0 & 11 & 0 & No \\
\hline 11 & 67 & 26 & Ascending colon & Moderately & 5 & $\mathrm{~T} 2$ & 3 & 0 & 11 & 1 & Yes \\
\hline 12 & 77 & 24 & Hepatic flexure & Poorly & 4.7 & $\mathrm{~T} 3$ & 2 & 1 & 16 & 1 & No \\
\hline 13 & 91 & 29 & Hepatic flexure & Moderately & 3.8 & $\mathrm{~T} 4$ & 4 & 2 & 18 & 9 & No \\
\hline 14 & 43 & 38 & Hepatic flexure & Moderately & 4 & $\mathrm{~T} 3$ & 3 & 0 & 11 & 0 & No \\
\hline 15 & 75 & 26 & Hepatic flexure & Moderately & 4.5 & $\mathrm{~T} 3$ & 2 & 0 & 19 & 0 & No \\
\hline 16 & 68 & 28 & Transverse colon & Moderately & 2.5 & $\mathrm{~T} 1$ & 3 & 2 & 16 & 6 & No \\
\hline 17 & 48 & 28 & Sigmoid & Well & 3 & $\mathrm{~T} 2$ & 5 & 1 & 10 & 2 & No \\
\hline 18 & 33 & 21 & Sigmoid & Moderately & 4 & $\mathrm{~T} 3$ & 3 & 0 & 18 & 0 & No \\
\hline 19 & 46 & 28 & Sigmoid & Moderately & 4.5 & $\mathrm{~T} 3$ & 3 & 0 & 14 & 0 & No \\
\hline 20 & 71 & 27 & Rectum & Poorly & 2.6 & $\mathrm{~T} 1$ & 2 & 0 & 8 & 0 & No \\
\hline 21 & 25 & 22 & Rectum & Moderately & 5.5 & $\mathrm{~T} 3$ & 2 & 2 & 24 & 5 & No \\
\hline 22 & 76 & 21 & Rectum & Minimal residual foci ${ }^{\mathrm{a}}$ & $\mathrm{n} / \mathrm{a}^{\mathrm{a}}$ & $\mathrm{T} 2$ & 1 & 0 & 14 & 0 & No \\
\hline 23 & 52 & 24 & Rectum & Moderately & 3 & $\mathrm{~T} 2$ & 5 & 0 & 17 & 0 & No \\
\hline 24 & 46 & 29 & Rectum & Moderately & 2 & $\mathrm{~T} 3$ & 2 & 1 & 10 & 1 & No \\
\hline Mean & 62.7 & 26.9 & & & 4.0 & & 3.0 & & 15.9 & & \\
\hline
\end{tabular}

$S L N$ sentinel lymph node, $N I R$ near-infrared, $L N$ lymph node, $L N+$ lymph node with histologically proven metastases

Tumor stage was determined by TNM classification

${ }^{a}$ Patient received neoadjuvant radiation and chemotherapy with a near-complete pathological response

According to the WHO Classification, 6th edition, such tumor deposits are regarded as lymph node involvement. This is supported by the configuration of the lesion, being encapsulated and round. Since the lesion was completely effaced by tumor cells and lymphatic tissue was not observable, it is plausible that no lymphatic drainage could reach this node and in fact the NIR lymphatic tracer had bypassed this mass. This phenomenon of lymph node effacement is well known for breast cancer patients. The fact that no fluorescence could be measured in the paraffin block of the lesion supports this hypothesis.

The great advantage of NIR fluorescence imaging is that the lymphatic channels traveling to the SLN are clearly visualized in real time, even deep within the bowel mesentery. NIR fluorescence imaging also permits the use of small volumes $(1 \mathrm{cc})$ of a colorless dye, and does not alter the appearance of the surgical field. Furthermore, the timing constraints of the technique we describe are very flexible, with successful identification of the SLN up to $1 \mathrm{~h}$ after resection of the bowel.

These results and earlier findings suggest that NIR imaging has the potential to simplify the SLN procedure in colorectal cancer, when compared with the use of visible contrast agents such as isosulfan blue. ${ }^{30,31}$ Therefore, larger studies comparing both techniques should be performed. The ex vivo approach has certain advantages, such as the use of contrast agents that are not yet approved for in vivo administration. ${ }^{11}$ The agent used in our study, HSA800, has previously been compared with clinically available agents such as indocyanine green and methylene blue and has clear advantages over them. ${ }^{30}$ Because of conjugation of fluorophore IRDye $800 \mathrm{CW}$ to human serum albumin (HSA800), the hydrodynamic diameter of the probe increases from less than 1 to $7.4 \mathrm{~nm}$, thereby facilitating a better retention in the first (sentinel) node compared with blue dyes. 

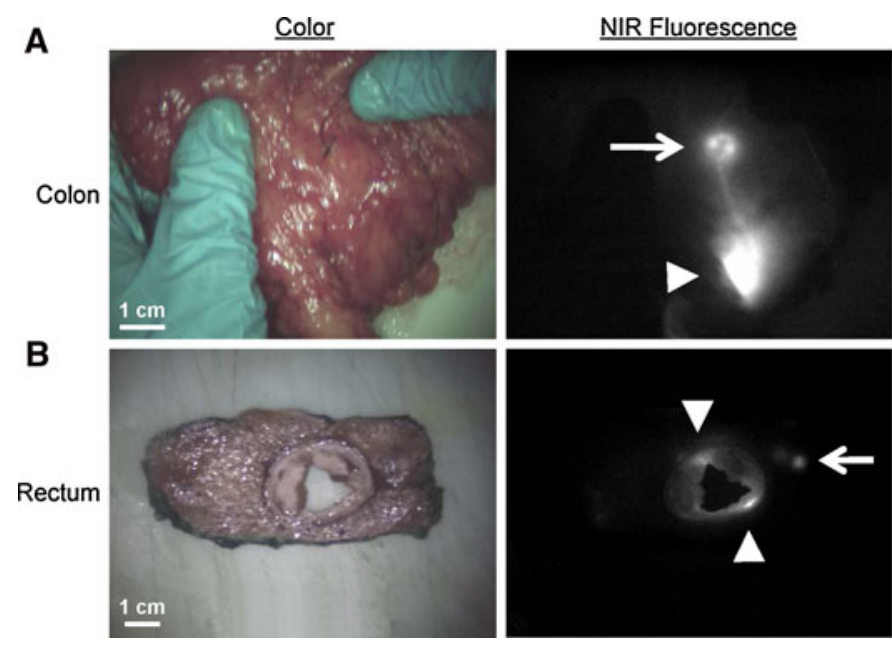
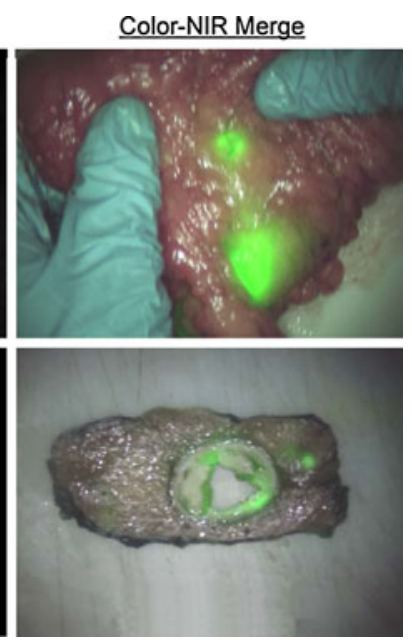

FIG. 3 NIR fluorescence-guided SLN mapping in patients with colorectal cancer. a Ex vivo identification of a SLN (arrow) in the mesentery of the right hemicolon of a patient with a cecal adenocarcinoma after submucosal peritumoral injection (arrowhead) of $1 \mathrm{cc}$ of $50 \mu \mathrm{M}$ HSA800. Shown are the color video (left), NIR fluorescence (middle), and pseudocolored (lime green) merge of the 2

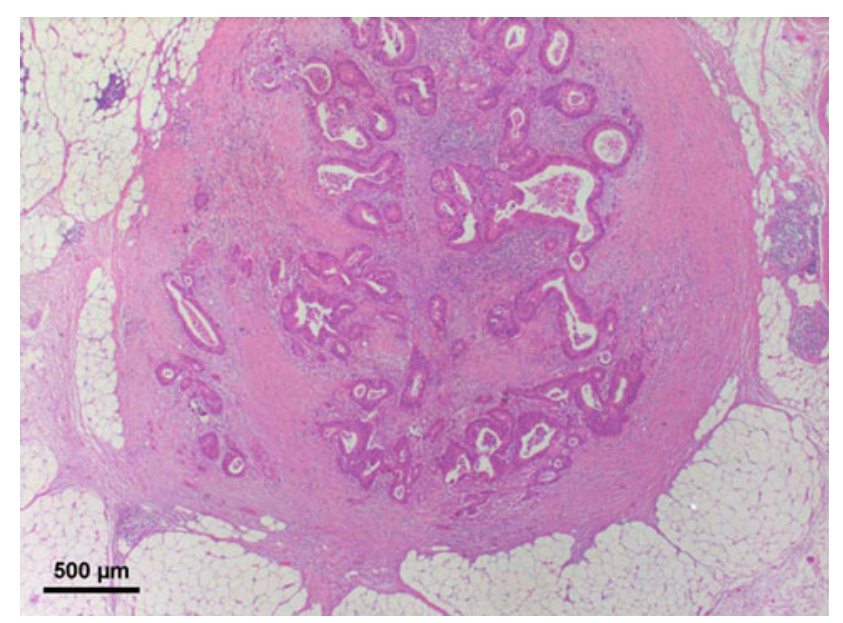

FIG. 4 Palpable metastatic lesion identified in the mesenteric fat. $\mathrm{H} \& \mathrm{E}$ histological staining of a mesenteric metastasis $(25 \times$ magnification) not identified as a SLN. No nodal tissue was observed

Because the agent is not injected into the patient, adverse reactions and patient safety are non-issues. Also, because the injection is performed after resection, it is not necessary that the surgeon performs the injection. Therefore, this technique does not add any time to the surgery, which is beneficial for the patient and could improve acceptance of the technique. In our hospital, the fluorescent dye is injected immediately after surgery at the department of pathology, thereby eliminating any interference with the surgical procedure. Using this technology, it should now be possible to design even more effective lymphatic tracers having higher fluorescence intensity and better lymph node retention. (right). b Ex vivo identification of a SLN (arrow) in the mesorectum of a patient with a rectal adenocarcinoma after submucosal peritumoral injection (arrowhead) of $1 \mathrm{cc}$ of $50 \mu \mathrm{M}$ HSA800. After $48 \mathrm{~h}$ fixation in $2 \%$ buffered formalin, tissue specimen was sliced and imaged. Shown are the color video (left), NIR fluorescence (middle), and pseudocolored (lime green) merge of the 2 (right)

The current NIR fluorescence imaging technique does not interfere with routine gross pathology and makes it possible to detect the SLN in sliced sections of rectal cancer specimens. Although neoadjuvant radiotherapy is known to affect lymphatic channels, even in rectal cancer patients that were treated with radiotherapy, SLNs were identified. A possible explanation is that only small amounts of HSA800 need to accumulate in the lymph node to be detected by the Mini-FLARE imaging system. As the mesorectum was not altered in any way, no lymphatic channels were disrupted. Furthermore, because lymph flow is induced by massaging the ex vivo specimen, decreased lymphatic flow can be overcome.

Compared with published clinical studies on SLN imaging using blue dyes, an important difference with the current study is the condition of the injection site. The current preclinical study was performed in healthy pigs, whereas the clinical application of this technique was performed in cancer patients. Large tumors are especially known to alter and disrupt lymphatic channels. Since earlier studies show that there is still sufficient lymphatic flow to identify lymph nodes, we did not expect this to be a major problem. ${ }^{13,39}$ Viehl and colleagues showed that with larger tumors, a larger amount of blue dye $(0.5 \mathrm{cc}$ per $\mathrm{cm}$ tumor diameter) should be used to identify the SLN. ${ }^{39}$ This observation suggests an advantage for NIR fluorescence imaging, since in our pilot clinical study in which the mean diameter of the tumors was $4.5 \mathrm{~cm}$, a smaller amount of dye $(1 \mathrm{cc})$ was sufficient to identify a fluorescent signal in the SLN.

In conclusion, our study suggests that ex vivo SLN mapping using an optimal lymphatic tracer and invisible 
NIR fluorescent light permits real-time imaging of lymph flow and identification of the SLN in colon and rectal cancer specimens.

ACKNOWLEDGMENT We thank Rita G. Laurence for assistance with animal anesthesia, Summer L. Gibbs-Strauss for cryosectioning, Lorissa A. Moffitt and Eugenia Trabucchi for administrative assistance, and Daan J. Lips, Jappe R. Licht, and Tjalling Bosse for technical assistance. This work was supported in part by NIH grant R01-CA-115296 (JVF), the Michäel van Vloten fund (AV), Foundation "De Drie Lichten" (MH), the Dutch Cancer Society (MH), Leiden University Fund $(\mathrm{MH})$, Nuts Ohra Fund, Foundation "Maurits and Anna de Kock," and the Center for Translational Molecular Medicine (DeCoDe project, grant 03O-101).

CONFLICT OF INTEREST All FLARE ${ }^{\mathrm{TM}}$ technology is owned by Beth Israel Deaconess Medical Center, a teaching hospital of Harvard Medical School. As inventor, Dr. Frangioni may someday receive royalties if products are commercialized. Dr. Frangioni is the founder and unpaid director of The FLARE Foundation, a non-profit organization focused on promoting the dissemination of medical imaging technology for research and clinical use.

OPEN ACCESS This article is distributed under the terms of the Creative Commons Attribution Noncommercial License which permits any noncommercial use, distribution, and reproduction in any medium, provided the original author(s) and source are credited.

\section{REFERENCES}

1. Giuliano AE, Dale PS, Turner RR, Morton DL, Evans SW, Krasne DL. Improved axillary staging of breast cancer with sentinel lymphadenectomy. Ann Surg. 1995;222:394-401.

2. Giuliano AE, Kirgan DM, Guenther JM, Morton DL. Lymphatic mapping and sentinel lymphadenectomy for breast cancer. Ann Surg. 1994;220:391-8.

3. Morton DL, Thompson JF, Essner R, Elashoff R, Stern SL, Nieweg OE, et al. Validation of the accuracy of intraoperative lymphatic mapping and sentinel lymphadenectomy for earlystage melanoma: a multicenter trial. Multicenter Selective Lymphadenectomy Trial Group. Ann Surg. 1999;230:453-63.

4. Adell G, Boeryd B, Frånlund B, Sjödahl R, Håkansson L. Occurrence and prognostic importance of micrometastases in regional lymph nodes in Dukes' B colorectal carcinoma: an immunohistochemical study. Eur J Surg. 1996;162:637-42.

5. Noura S, Yamamoto H, Ohnishi T, Masuda N, Matsumoto T, Takayama $\mathrm{O}$, et al. Comparative detection of lymph node micrometastases of stage II colorectal cancer by reverse transcriptase polymerase chain reaction and immunohistochemistry. J Clin Oncol. 2002;20:4232-41.

6. Palma RT, Waisberg J, Bromberg SH, Simão AB, Godoy AC. Micrometastasis in regional lymph nodes of extirpated colorectal carcinoma: immunohistochemical study using anti-cytokeratin antibodies AE1/AE3. Colorectal Dis. 2003;5:164-8.

7. Liefers GJ, Cleton-Jansen AM, van de Velde CJ, Hermans J, van Krieken JH, Cornelisse CJ, et al. Micrometastases and survival in stage II colorectal cancer. N Engl J Med. 1998;339:223-8.

8. Doekhie FS, Mesker WE, Kuppen PJ, van Leeuwen GA, Morreau $\mathrm{H}$, de Bock $\mathrm{GH}$, et al. Detailed examination of lymph nodes improves prognostication in colorectal cancer. Int $J$ Cancer. 2010;126:2644-52.
9. Bilchik AJ, Hoon DS, Saha S, Turner RR, Wiese D, DiNome M, et al. Prognostic impact of micrometastases in colon cancer: interim results of a prospective multicenter trial. Ann Surg. 2007;246:568-75.

10. Iddings D, Bilchik A. The biologic significance of micrometastatic disease and sentinel lymph node technology on colorectal cancer. J Surg Oncol. 2007;96:671-7.

11. Nordgård O, Oltedal S, Kørner H, Aasprong OG, Tjensvoll K, Gilje B, et al. Quantitative RT-PCR detection of tumor cells in sentinel lymph nodes isolated from colon cancer patients with an ex vivo approach. Ann Surg. 2009;249:602-7.

12. Saha S, Seghal R, Patel M, Doan K, Dan A, Bilchik A, et al. A multicenter trial of sentinel lymph node mapping in colorectal cancer: prognostic implications for nodal staging and recurrence. Am J Surg. 2006;191:305-10.

13. Stojadinovic A, Allen PJ, Protic M, Potter JF, Shriver CD, Nelson JM, et al. Colon sentinel lymph node mapping: practical surgical applications. J Am Coll Surg. 2005;201:297-313.

14. van der Zaag ES, Buskens CJ, Kooij N, Akol H, Peters HM, Bouma $\mathrm{WH}$, et al. Improving staging accuracy in colon and rectal cancer by sentinel lymph node mapping: a comparative study. Eur J Surg Oncol. 2010;35:1065-70.

15. Wiese D, Saha S, Yestrepsky B, Korant A, Sirop S. A prospective study of false-positive diagnosis of micrometastatic cells in the sentinel lymph nodes in colorectal cancer. Ann Surg Oncol. 2009; 16:2166-69.

16. Wong JH, Johnson DS, Namiki T, Tauchi-Nishi P. Validation of ex vivo lymphatic mapping in hematoxylin-eosin node-negative carcinoma of the colon and rectum. Ann Surg Oncol. 2004;11:772-7.

17. Wong JH, Steineman S, Calderia C, Bowles J, Namiki T. Ex vivo sentinel node mapping in carcinoma of the colon and rectum. Ann Surg. 2001;233:515-21.

18. Doekhie FS, Peeters KC, Kuppen PJ, Mesker WE, Tanke HJ, Morreau $\mathrm{H}$, et al. The feasibility and reliability of sentinel node mapping in colorectal cancer. Eur J Surg Oncol. 2005;31:854-62.

19. Bembenek AE, Rosenberg R, Wagler E, Gretschel S, Sendler A, Siewert JR, et al. Sentinel lymph node biopsy in colon cancer: a prospective multicenter trial. Ann Surg. 2007;245:858-63.

20. Bilchik AJ, DiNome M, Saha S, Turner RR, Wiese D, McCarter $\mathrm{M}$, et al. Prospective multicenter trial of staging adequacy in colon cancer: preliminary results. Arch Surg. 2006;141:527-33.

21. Stojadinovic A, Nissan A, Protic M, Adair CF, Prus D, Usaj S, et al. Prospective randomized study comparing sentinel lymph node evaluation with standard pathologic evaluation for the staging of colon carcinoma: results from the United States Military Cancer Institute Clinical Trials Group Study GI-01. Ann Surg. 2007;245:846-57.

22. Frangioni JV. In vivo near-infrared fluorescence imaging. Curr Opin Chem Biol. 2003;7:626-34.

23. Kitai T, Inomoto T, Miwa M, Shikayama T. Fluorescence navigation with indocyanine green for detecting sentinel lymph nodes in breast cancer. Breast Cancer. 2005;12:211-5.

24. Kusano M, Tajima Y, Yamazaki K, Kato M, Watanabe M, Miwa M. Sentinel node mapping guided by indocyanine green fluorescence imaging: a new method for sentinel node navigation surgery in gastrointestinal cancer. Dig Surg. 2008;25:103-8.

25. Miyashiro I, Miyoshi N, Hiratsuka M, Kishi K, Yamada T, Ohue $\mathrm{M}$, et al. Detection of sentinel node in gastric cancer surgery by indocyanine green fluorescence imaging: comparison with infrared imaging. Ann Surg Oncol. 2008;15:1640-3.

26. Ogasawara Y, Ikeda H, Takahashi M, Kawasaki K, Doihara H. Evaluation of breast lymphatic pathways with indocyanine green fluorescence imaging in patients with breast cancer. World $J$ Surg. 2008;32:1924-9. 
27. Sevick-Muraca EM, Sharma R, Rasmussen JC, Marshall MV, Wendt JA, Pham HQ, et al. Imaging of lymph flow in breast cancer patients after microdose administration of a near-infrared fluorophore: feasibility study. Radiology. 2008;246:734-41.

28. Tanaka E, Choi HS, Fujii H, Bawendi MG, Frangioni JV. Imageguided oncologic surgery using invisible light: completed preclinical development for sentinel lymph node mapping. Ann Surg Oncol. 2006;13:1671-81.

29. Troyan SL, Kianzad V, Gibbs-Strauss SL, Gioux S, Matsui A, Oketokoun R, et al. The FLARE intraoperative near-infrared fluorescence imaging system: a first-in-human clinical trial in breast cancer sentinel lymph node mapping. Ann Surg Oncol. 2009:16:2943-52.

30. Ohnishi S, Lomnes SJ, Laurence RG, Gogbashian A, Mariani G, Frangioni JV. Organic alternatives to quantum dots for intraoperative near-infrared fluorescent sentinel lymph node mapping. Mol Imaging. 2005;4:172-81.

31. Soltesz EG, Kim S, Kim SW, Laurence RG, De Grand AM, Parungo CP, et al. Sentinel lymph node mapping of the gastrointestinal tract by using invisible light. Ann Surg Oncol. 2006;13:386-96.

32. Kim S, Lim YT, Soltesz EG, De Grand AM, Lee J, Nakayama A, et al. Near-infrared fluorescent type II quantum dots for sentinel lymph node mapping. Nat Biotechnol. 2004;22:93-7.

33. Quirke P, Durdey P, Dixon MF, Williams NS. Local recurrence of rectal adenocarcinoma due to inadequate surgical resection.
Histopathological study of lateral tumour spread and surgical excision. Lancet. 1986;2:996-9.

34. van Schaik PM, van der Linden JC, Ernst MF, Gelderman WA, Bosscha K. Ex vivo sentinel lymph node "mapping" in colorectal cancer. Eur J Surg Oncol. 2007;33:1177-82.

35. International Multicentre Pooled Analysis of Colon Cancer Trials (IMPACT) investigators. Efficacy of adjuvant fluorouracil and folinic acid in colon cancer. Lancet. 1995;345:939-44.

36. Wolmark N, Rockette H, Fisher B, Wickerham DL, Redmond C, Fisher ER, et al. The benefit of leucovorin-modulated fluorouracil as postoperative adjuvant therapy for primary colon cancer: results from National Surgical Adjuvant Breast and Bowel Project protocol C-03. J Clin Oncol. 1993;11:1879-87.

37. Bertagnolli M, Miedema B, Redston M, Dowell J, Niedzwiecki D, Fleshman J, et al. Sentinel node staging of resectable colon cancer: results of a multicenter study. Ann Surg. 2004;240: 624-30.

38. Redston M, Compton CC, Miedema BW, Niedzwiecki D, Dowell JM, Jewell SD, et al. Analysis of micrometastatic disease in sentinel lymph nodes from resectable colon cancer: results of Cancer and Leukemia Group B Trial 80001. J Clin Oncol. 2006;24:878-83.

39. Viehl CT, Hamel CT, Marti WR, Guller U, Eisner L, Stammberger $\mathrm{U}$, et al. Identification of sentinel lymph nodes in colon cancer depends on the amount of dye injected relative to tumor size. World J Surg. 2003;27:1285-90. 